\title{
Epidemiologia do Uso de Internet numa População Adolescente e Sua Relação com Hábitos de Sono
}

\author{
Epidemiology of Internet Use by an Adolescent \\ Population and its Relation with Sleep Habits
}

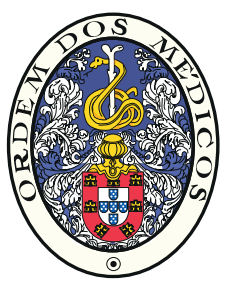

\author{
Carla FERREIRA $\otimes^{1}$, Helena FERREIRA ${ }^{1}$, Maria João VIEIRA'1, Mónica COSTEIRA ${ }^{1}$, Liliana BRANCO ${ }^{1}$, Ângela DIAS ${ }^{1}$, \\ Liliana MACEDO 1 \\ Acta Med Port 2017 Jul-Aug;30(7-8):524-533 - https://doi.org/10.20344/amp.8205
}

\section{RESUMO}

Introdução: A grande evolução tecnológica das últimas décadas tornou a Internet cada vez mais popular, sendo crescente a preocupação com a sua utilização excessiva. Foram objetivos do presente trabalho aferir e caracterizar o uso de Internet na adolescência, determinar a dependência de Internet e estabelecer a sua associação com alterações do sono e sonolência diurna excessiva.

Material e Métodos: Foi realizado um estudo de base comunitária, observacional e transversal dirigido a adolescentes a frequentar o sétimo e oitavo ano de escolaridade. Recorreu-se a um questionário de autopreenchimento online, para aferir características sociodemográficas, uso e dependência de Internet, características do sono e sonolência diurna excessiva.

Resultados: Foram incluídos 727 adolescentes com idade média de $13 \pm 0,9$ anos. Três quartos dos adolescentes usa a Internet diariamente e $41 \%$ fá-lo durante três ou mais horas/dia, maioritariamente em casa. O telemóvel e o computador portátil são os principais dispositivos utilizados. Os jogos online e o uso das redes sociais são das principais atividades realizadas. A dependência de Internet foi observada em $19 \%$ dos adolescentes, associando-se ao género masculino, à utilização de redes sociais, o Twitter e Instagram, à autopercepção de problemas de sono, insónia inicial e intermédia e sonolência diurna excessiva $(p<0,05)$.

Discussão: Os resultados reiteram o destaque que a Internet tem na rotina dos adolescentes, que priorizam o seu uso no acesso a redes sociais e jogos online, fazendo-o com dispositivos de uso individual, menos passíveis de controlo parental.

Conclusão: A dependência de Internet verificada e associação com alterações do sono e sonolência diurna excessiva enfatiza a relevância desta problemática.

Palavras-chave: Adolescente; Comportamento Aditivo; Distúrbios do Sono por Sonolência Excessiva; Internet; Social Media; Sono

\section{ABSTRACT}

Introduction: In the last decades, the great technological development increased Internet popularity, emerging the concern about its overuse. The objectives of this study were to assess and characterize Internet use in adolescence, determine Internet addiction and clarify its association with sleep disorders and excessive daytime sleepiness.

Material and Methods: It was performed an observational, cross sectional and community-based study. The target were students attending $7^{\text {th }}$ and $8^{\text {th }}$ grades, to whom was applied an online self-report questionnaire to assess sociodemographic features, Internet use, Internet dependence, sleep characteristics and excessive daytime sleepiness.

Results: A total of 727 adolescents were included with a mean age $13 \pm 0.9$ years. Three-quarters of teenagers use Internet daily and $41 \%$ do it for three or more hours/day, mainly at home. The phone and laptop were the main devices used. Online games and social networks use were the main activities performed. Internet dependence was observed in $19 \%$ of adolescents, and it was associated with male gender, social networks use, mainly Twitter and Instagram use, self-perceived sleep problems, initial and middle insomnia and excessive daytime sleepiness $(p<0.05)$.

Discussion: The results confirm the highlight that Internet has in adolescents routine, who prioritize in their use access to social networks and online games, using single devices, less subject to parental control.

Conclusion: The Internet addiction rate observed and its association with sleep alterations and daytime sleepiness emphasizes the importance of this issue.

Keywords: Adolescent; Behavior, Addictive; Disorders of Excessive Somnolence; Internet; Sleep; Social Media

\section{INTRODUÇÃO}

A grande evolução tecnológica das últimas décadas tornou a Internet cada vez mais popular, fazendo atualmente parte integrante do quotidiano da sociedade moderna, como meio de informação, diversão e socialização. Os seus principais utilizadores são adolescentes e jovens adultos, estimando-se que $70 \%$ dos adolescentes europeus disponham de 2 a 4 horas do seu dia em atividades online.$^{1-3} \mathrm{~A}$ utilização de Internet cada vez mais frequente tem sido tema de debate, pois questiona-se qual será o limite aceitável de utilização e quais as consequências do uso excessivo no desenvolvimento e saúde dos jovens utilizadores. ${ }^{4}$ Foi em 1996 que Kimberly Young utilizou pela primeira vez o termo
'Internet addiction'. ${ }^{5}$ Desde então, diferentes terminologias têm sido utilizadas para descrever a utilização excessiva e compulsiva de Internet, com consequências negativas a nível pessoal, profissional ou social. De acordo com as diferentes escalas aplicadas, diferentes definições são utilizadas, como dependência de Internet, uso excessivo, problemático ou patológico de Internet. ${ }^{6}$ Atualmente, das várias escalas disponíveis, a Internet Addiction Test (IAT) é das mais utilizadas. ${ }^{7-11}$ Trata-se de um questionário cujo somatório de 20 questões permite determinar se o adolescente não tem dependência de Internet, ou por outro lado se tem dependência de Internet, podendo ser moderada ou grave.

1. Serviço de Pediatria. Hospital da Senhora da Oliveira. Guimarães. Portugal.

$\triangle$ Autor correspondente: Carla Ferreira. carlamf85@hotmail.com

Recebido: 11 de setembro de 2016 - Aceite: 10 de abril de 2017 | Copyright @ Ordem dos Médicos 2017 
A aplicação da IAT a 475 adolescentes finlandeses permitiu aferir que um quarto dos inquiridos apresentava critérios de dependência de Internet moderada a grave. ${ }^{12}$ Segundo Milani et al, 36,7\% dos adolescentes italianos apresentavam sinais de uso problemático de Internet e a sua utilização excessiva esteve associada a estratégias de coping disfuncional e piores relações interpessoais. ${ }^{13}$ De facto, vários estudos demonstram o impacto negativo do uso excessivo de Internet sobre o desenvolvimento psicossocial dos adolescentes, ${ }^{14-16}$ assim como a sua associação com comportamentos suicidas (ideação suicida e tentativas de suicídio), depressão e ansiedade. ${ }^{17-19}$ No que diz respeito a alterações do sono existem algumas associações positivas descritas, mas do conhecimento dos autores não existe, até à data, nenhum estudo nacional sobre a prevalência de dependência de Internet na adolescência, nem sobre o seu efeito no sono e na sonolência diurna. ${ }^{20-24}$ Assim, foram objetivos principais do presente estudo: (1) aferir e caracterizar o uso de Internet na adolescência; (2) determinar a dependência de Internet na adolescência; (3) avaliar a associação entre a dependência de Internet e alterações do sono/sonolência diurna excessiva.

\section{MATERIAL E MÉTODOS \\ Desenho do estudo}

Procedeu-se à realização de um estudo de base comunitária, observacional, transversal e analítico dirigido a adolescentes dos sétimos e oitavos anos de escolaridade. Fo- ram selecionados todos os agrupamentos escolares de um concelho do Norte do país, com elevada densidade populacional e incluídos os seis que aceitaram participar. Os agrupamentos de escolas incluídos foram agrupamentos predominantemente urbanos, localizados em cidades ou vilas. A recolha de informação foi realizada através da aplicação de um questionário eletrónico de autopreenchimento, anónimo e confidencial, com preenchimento obrigatório de todas as questões. O questionário foi preenchido de forma individual na aula de Tecnologia de Informação e Comunicação (TIC), durante o mês de Abril de 2016. O estudo foi aprovado pela comissão de ética e foi obtido o consentimento informado do representante legal dos participantes.

\section{Recolha de informação}

O questionário, composto por 67 questões fechadas, foi organizado em três partes distintas (Tabela 1).

\section{Análise dos dados}

As variáveis categóricas foram apresentadas em frequências e percentagens e as variáveis contínuas em média e desvio padrão. Foram utilizados na comparação de variáveis categóricas os testes de qui-quadrado ou o teste exato de Fisher (quando adequado) e na comparação de varáveis contínuas foi utilizado o $t$-student. Recorreu-se ao programa informático Statistical Package for the Social Sciences ${ }^{\circledR}$ (SPSS), versão 22.0 e foi admitido significado estatístico para valores de $p<0,05$.

Tabela 1 - Questões abordadas no questionário

\section{Caracterização sociodemográfica e características pessoais do adolescente}

1.1. Características do adolescente

Idade; género; número de reprovações; prática extracurricular de desporto regular; atividades de grupo extracurriculares.

1.2. Características da família

Tipo de família; idades dos pais; habilitações literárias dos pais.

\section{Caracterização do uso de Internet e dependência de Internet}

\subsection{Caracterização do uso de Internet}

Acesso prévio à Internet; idade da primeira utilização de Internet; frequência de acesso à Internet; número de horas diárias utilizadas em atividades online; dispositivo utilizados no acesso à Internet; local de acesso; motivos de acesso; atividades realizadas online; frequência de uso de jogos online; número de horas diárias utilizadas em jogos online; frequência de uso de RS; número de horas diárias utilizadas em RS; tipo de RS utilizadas; configuração de privacidade do perfil da RS; atitude face a pedidos de amizade de desconhecidos; partilha de fotografias pessoais nas RS; divulgação da localização nas RS; divulgação de contactos pessoais e morada nas RS; encontros com conhecidos exclusivamente das RS; conhecimento dos pais/ responsáveis sobre atividades realizadas online; cumprimento das orientações parentais sobre o uso da Internet.

\subsection{Dependência de Internet}

Para definir dependência de Internet utilizou-se o IAT, um questionário validado para a população portuguesa cujo somatório de 20 questões permitiu classificar o adolescente como tendo dependência moderada (50 a 79 pontos) ou dependência grave de Internet (superior a 80 pontos). ${ }^{25}$

\section{Caracterização dos hábitos de sono e sonolência diurna excessiva}

3.1. Caracterização dos hábitos de sono

Autopercepção de problemas de sono; insónias inicial, intermédia e terminal; pesadelos; realização de sesta; número de horas de sono.

\subsection{Sonolência diurna excessiva}

Foi usado o PDSS (Pediatric Daytime Sleepiness Scale), instrumento validado para população a portuguesa, com oito questões, cuja cotação superior a 20 pontos indicou existência de sonolência diurna excessiva. ${ }^{26}$ 


\section{RESULTADOS}

\section{Caracterização da amostra}

Foram incluídos 727 adolescentes, de um total de 2345 inscritos em todos os agrupamentos de escolas do concelho. A idade média foi $13 \pm 0,9$ anos e observou-se um discreto predomínio do género masculino (53\%). A prática de atividades extracurriculares, nomeadamente atividade física regular foi referida por $60 \%$ dos adolescentes e atividades de grupo por $41 \%$ (Tabela 2). No que diz respeito às características da família, as médias de idade da mãe e do pai foram $42 \pm 5$ anos e $46 \pm 6$ anos, respetivamente. $A$ maioria $(76 \%)$ fazia parte de uma família nuclear (Tabela 2 ).

\section{Caracterização do uso de Internet}

Todos os adolescentes referiram utilização prévia de Internet e $28 \%$ admitiu tê-lo feito pela primeira vez com menos de seis anos de idade. A utilização de Internet foi realizada principalmente em casa e em locais públicos gratuitos, através do telemóvel, portátil e tablet. Três quartos da amostra utilizou a Internet diariamente e $41 \%$ fê-lo três ou mais horas por dia. Diversão, comunicação e socialização foram os principais motivos de acesso à Internet, sendo os jogos online e a utilização de RS as principais atividades realizadas. Três ou mais horas por dia foi o tempo despendido a jogar online e usar RS por $24 \%$ e $31 \%$ dos adoles- centes, respetivamente (Tabela 3).

As principais RS utilizadas foram o Facebook e Instagram e $57 \%$ usou uma configuração de perfil privada. A maioria utilizou as RS para comunicar com amigos reais e $17 \%$ admitiu ter-se encontrado com amigos virtuais (Tabela 3). A publicação de fotografias pessoais foi a principal forma de partilha de informação pessoal realizada nas RS, raramente foi divulgada a localização, a morada e os contactos pessoais (Fig. 1). O conhecimento parental das atividades realizadas online foi classificado como muito bom por $52 \%$ dos adolescentes e pouco mais de metade admitiu ter seguido com frequência as recomendações parentais relativas à utilização de Internet (Tabela 3 ).

\section{Caracterização dos hábitos de sono}

Um em cada dez adolescentes reportou problemas de sono, tendo sido a insónia intermédia $(41 \%)$ a mais frequente. Cerca de metade dos adolescentes referiu dormir menos de nove horas diárias. Tinha critério de sonolência diurna excessiva $11 \%$ da amostra (Tabela 4).

\section{Dependência de Internet}

Verificou-se critérios de dependência de Internet em $19 \%$ da amostra, dos quais $2 \%$ tinham critérios de dependência grave (Tabela 3 ). Apenas o género masculino

Tabela 2 - Características sociodemográficas do adolescente e da sua família ( $n=727$ )

\begin{tabular}{|c|c|c|}
\hline Características & & $\%(n)$ \\
\hline \multicolumn{3}{|l|}{ Características do adolescente } \\
\hline \multirow{2}{*}{ Género } & Masculino & $53(386)$ \\
\hline & Feminino & $47(341)$ \\
\hline \multirow{2}{*}{ Reprovações prévias } & Não & $80(584)$ \\
\hline & Sim & $20(143)$ \\
\hline \multirow{2}{*}{ Prática extracurricular de desporto regular } & Não & $40(294)$ \\
\hline & Sim & $60(433)$ \\
\hline \multirow{2}{*}{ Atividades de grupo extracurriculares } & Não & $59(430)$ \\
\hline & Sim & $41(297)$ \\
\hline \multicolumn{3}{|l|}{ Características da família } \\
\hline \multirow{4}{*}{ Tipo de família } & Nuclear & $76(553)$ \\
\hline & Alargada & $11(78)$ \\
\hline & Monoparental & $11(78)$ \\
\hline & Outra & $2(18)$ \\
\hline \multirow{5}{*}{ Habilitações literárias da mãe } & $\leq 4^{\circ}$ ano & $18(131)$ \\
\hline & $6^{\circ}$ ano & $28(204)$ \\
\hline & $9^{\circ}$ ano & $23(167)$ \\
\hline & $12^{\circ}$ ano & $19(138)$ \\
\hline & Licenciatura & $12(87)$ \\
\hline \multirow{5}{*}{ Habilitações literárias do pai } & $\leq 4^{\circ}$ ano & $22(160)$ \\
\hline & $6^{\circ}$ ano & $29(211)$ \\
\hline & $9^{\circ}$ ano & $22(160)$ \\
\hline & $12^{\circ}$ ano & $20(145)$ \\
\hline & Licenciatura & $7(51)$ \\
\hline
\end{tabular}


Tabela 3 - Caracterização do uso de Internet e dependência de Internet (parte 1 de 2)

\begin{tabular}{|c|c|c|}
\hline \multicolumn{2}{|l|}{ Características } & \multirow[t]{2}{*}{$\%(n)$} \\
\hline Uso de Internet $(n=727)$ & & \\
\hline \multirow{2}{*}{ Acesso prévio à Internet } & Não & $0(0)$ \\
\hline & Sim & $100(727)$ \\
\hline \multirow{8}{*}{ Idade da primeira utilização } & $\leq 6$ anos & $28(203)$ \\
\hline & 7 anos & $16(120)$ \\
\hline & 8 anos & $11(77)$ \\
\hline & 9 anos & $11(76)$ \\
\hline & 10 anos & $11(77)$ \\
\hline & 11 anos & $2(17)$ \\
\hline & 12 anos & $1(13)$ \\
\hline & Desconhece & $20(144)$ \\
\hline \multirow{4}{*}{ Frequência de utilização de Internet } & $<1 \mathrm{vez} / \mathrm{semana}$ & $5(36)$ \\
\hline & 1 - 3 vezes/semana & $10(76)$ \\
\hline & 4 - 6 vezes/semana & $10(71)$ \\
\hline & Diariamente & $75(544)$ \\
\hline \multirow{4}{*}{ Número de horas diárias utilizadas em atividades online } & $<1$ hora & $20(147)$ \\
\hline & 1 - 2 horas & $39(285)$ \\
\hline & $3-6$ horas & $31(227)$ \\
\hline & $>6$ horas & $10(68)$ \\
\hline \multirow{6}{*}{ Dispositivo utilizado para acesso à Internet } & Telemóvel & $94(684)$ \\
\hline & Computador & $30(218)$ \\
\hline & Portátil & $73(531)$ \\
\hline & Tablet & $59(429)$ \\
\hline & Televisão & $21(153)$ \\
\hline & Consola & $18(131)$ \\
\hline \multirow{7}{*}{ Local/meio de acesso à Internet } & Internet móvel própria & $34(247)$ \\
\hline & Internet móvel gratuita & $26(189)$ \\
\hline & Casa & $94(684)$ \\
\hline & Escola & $51(370)$ \\
\hline & Locais públicos gratuitos & $64(465)$ \\
\hline & Locais pagos & $3(22)$ \\
\hline & Casa de outros & $60(436)$ \\
\hline \multirow{5}{*}{ Motivo de acesso à Internet } & Diversão & $82(596)$ \\
\hline & Comunicação & $80(582)$ \\
\hline & Socialização & $77(560)$ \\
\hline & Motivos escolares & $52(378)$ \\
\hline & Pesquisa de informação & $51(371)$ \\
\hline \multirow{10}{*}{ Atividades realizadas online } & Ver filmes/séries/vídeos & $74(538)$ \\
\hline & Ver televisão & $21(153)$ \\
\hline & Fazer downloads & $48(349)$ \\
\hline & Ler revistas/livros & $7(51)$ \\
\hline & Ouvir música & $66(480)$ \\
\hline & Jogar online & $65(474)$ \\
\hline & Comunicar com amigos & $80(582)$ \\
\hline & Utilizar e-mail & $38(276)$ \\
\hline & $\mathrm{RS}$ & $94(680)$ \\
\hline & Realizar chamadas & $45(327)$ \\
\hline \multirow{3}{*}{$\begin{array}{l}\text { Conhecimento dos pais/responsáveis sobre atividades } \\
\text { realizadas pelo adolescente na Internet }\end{array}$} & Nada/pouco & $15(109)$ \\
\hline & Razoável & $33(240)$ \\
\hline & Bom/muito bom & $52(378)$ \\
\hline \multirow{3}{*}{$\begin{array}{l}\text { Cumprimento das orientações dos pais/responsáveis sobre o } \\
\text { uso da Internet }\end{array}$} & Nunca/raramente & $5(36)$ \\
\hline & Ocasionalmente & $40(291)$ \\
\hline & Muitas vezes/sempre & $55(400)$ \\
\hline
\end{tabular}

esteve associado a dependência $(p<0,05)$ (Tabela 5). Das cos gratuitos, na escola, para utilizar RS, nomeadamente o características familiares avaliadas, nenhuma esteve asso- Twitter e o Instagram esteve associado a dependência de ciada à presença de dependência de Internet (Tabela 5). Internet $(p<0,05)$ (Tabela 6$)$.

A utilização de Internet móvel própria, em locais públi- 
Tabela 3 - Caracterização do uso de Internet e dependência de Internet (parte 2 de 2)

\begin{tabular}{|c|c|c|}
\hline Características & & $\%(n)$ \\
\hline \multicolumn{3}{|l|}{ Jogos online $(n=474)$} \\
\hline \multirow{4}{*}{ Frequência de utilização de jogos online } & $<1 \mathrm{vez} /$ semana & $24(114)$ \\
\hline & 1 - 3 vezes/semana & $26(123)$ \\
\hline & 4 - 6 vezes/semana & $16(76)$ \\
\hline & Diariamente & $34(161)$ \\
\hline \multirow{4}{*}{ Número de horas diárias utilizadas em jogos online } & $<1$ hora & $38(180)$ \\
\hline & $1-2$ horas & $38(180)$ \\
\hline & $3-6$ horas & $18(85)$ \\
\hline & $>6$ horas & $6(28)$ \\
\hline \multicolumn{3}{|l|}{$\operatorname{RS}(n=680)$} \\
\hline \multirow{4}{*}{ Frequência de utilização de RS } & $<1 \mathrm{vez} /$ semana & $8(54)$ \\
\hline & 1 - 3 vezes/semana & $15(102)$ \\
\hline & 4 - 6 vezes/semana & $10(68)$ \\
\hline & Diariamente & $67(456)$ \\
\hline \multirow{4}{*}{ Número de horas diárias utilizadas na RS } & $<1$ hora & $34(231)$ \\
\hline & $1-2$ horas & $35(238)$ \\
\hline & $3-6$ horas & $21(143)$ \\
\hline & $>6$ horas & $10(68)$ \\
\hline \multirow{5}{*}{ RS utilizadas } & Facebook & $74(503)$ \\
\hline & Twitter & $22(150)$ \\
\hline & Snapchat & $69(469)$ \\
\hline & Instagram & $77(524)$ \\
\hline & Outras & $10(68)$ \\
\hline \multirow{3}{*}{ Configuração de privacidade do perfil da RS } & Público & $34(231)$ \\
\hline & Privado & $57(388)$ \\
\hline & Parcialmente privado & $9(61)$ \\
\hline \multirow{3}{*}{ Tipo de amigos com que o adolescente comunica nas RS } & Amigos reais & $80(544)$ \\
\hline & Amigos virtuais & $2(14)$ \\
\hline & Ambos & $18(122)$ \\
\hline \multirow{2}{*}{ Encontro com amigos conhecidos exclusivamente na RS } & Não & $83(564)$ \\
\hline & Sim & $17(116)$ \\
\hline \multicolumn{3}{|l|}{ Dependência de Internet $(n=727)$} \\
\hline \multirow{3}{*}{ AIT } & Sem dependência & $81(589)$ \\
\hline & Dependência moderada & $17(124)$ \\
\hline & Dependência grave & $2(14)$ \\
\hline
\end{tabular}

No que diz respeito aos hábitos de sono, a autopercepção de problemas de sono, presença de insónia inicial, insónia intermedia e dormir menos de nove horas por dia associaram-se a dependência de Internet $(p<0,05)$ (Tabela 4). Apresentar critérios de sonolência diurna excessiva também esteve associada a dependência de Internet $(p<$ 0,05) (Tabela 4).

\section{DISCUSSÃo}

Os resultados obtidos reiteram o destaque que a Internet

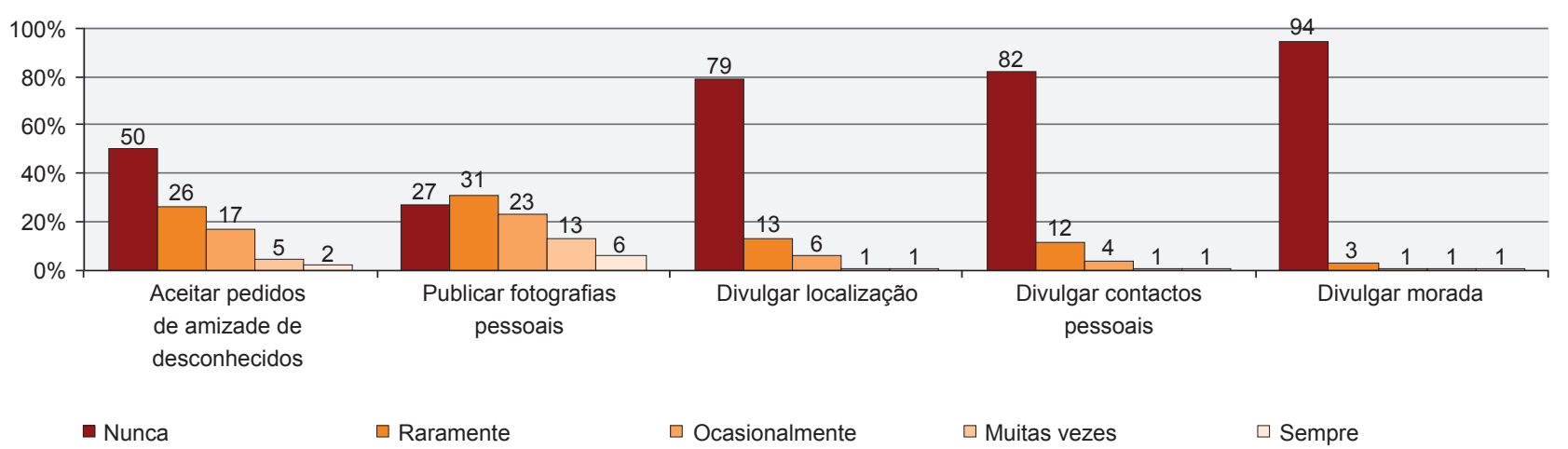

Figura 1 - Segurança das atividades realizadas pelos adolescentes nas RS

Segurança das atividades realizadas pelos adolescentes quando utilizam as redes sociais, nomeadamente a frequência com que aceitam pedidos de amizade de desconhecidos nas RS, publicação de fotografias pessoais, divulgação de localização contactos pessoais e morada. 
Tabela 4 - Caracterização dos hábitos de sono e sonolência diurna da amostra e caracterização dos hábitos de sono e sonolência diurna de acordo com a existência ou não de dependência de Internet

\begin{tabular}{|c|c|c|c|c|c|c|}
\hline \multicolumn{2}{|l|}{ Características } & $\begin{array}{c}\text { Todos } \\
\% \\
\end{array}$ & $\begin{array}{c}\text { Sem dependência } \\
\text { Internet } \\
\%(n)\end{array}$ & \multirow{2}{*}{$\begin{array}{l}\text { Com dependência } \\
\text { Internet } \\
\%(n)\end{array}$} & \multirow[t]{2}{*}{$\chi^{2}(\mathrm{df})$} & \multirow[t]{2}{*}{$p$} \\
\hline \multicolumn{4}{|l|}{ Hábitos de sono } & & & \\
\hline \multirow{2}{*}{$\begin{array}{l}\text { Autopercepção de problemas de } \\
\text { sono }\end{array}$} & Não & 90 & $83(519)$ & $17(105)$ & \multirow{2}{*}{$\begin{array}{l}25,478 \\
(1)\end{array}$} & \multirow{2}{*}{0,000} \\
\hline & Sim & 10 & $58(42)$ & $42(30)$ & & \\
\hline \multirow{2}{*}{ Insónia inicial } & Não & 82 & $84(483)$ & $16(90)$ & \multirow{2}{*}{$\begin{array}{l}28,234 \\
(1)\end{array}$} & \multirow{2}{*}{0,000} \\
\hline & $\operatorname{Sim}$ & 18 & $63(78)$ & $37(45)$ & & \\
\hline \multirow{2}{*}{ Insónia intermédia } & Não & 59 & $83(346)$ & $17(69)$ & \multirow{2}{*}{$\begin{array}{l}5,045 \\
(1)\end{array}$} & \multirow{2}{*}{0,025} \\
\hline & $\operatorname{Sim}$ & 41 & 76 ( 215) & $24(66)$ & & \\
\hline \multirow{2}{*}{ Insónia terminal } & Não & 69 & $82(394)$ & $18(89)$ & \multirow{2}{*}{$\begin{array}{l}0,950 \\
(1)\end{array}$} & \multirow{2}{*}{0,330} \\
\hline & $\operatorname{Sim}$ & 31 & 78 (167) & $22(46)$ & & \\
\hline \multirow{2}{*}{ Pesadelos } & Não & 88 & $81(502)$ & $19(114)$ & \multirow{2}{*}{$\begin{array}{l}2,716 \\
(1)\end{array}$} & \multirow{2}{*}{0,099} \\
\hline & Sim & 12 & $74(59)$ & $26(21)$ & & \\
\hline \multirow{2}{*}{ Sesta } & Não & 88 & $82(500)$ & $18(113)$ & \multirow{2}{*}{$\begin{array}{l}3,047 \\
(1)\end{array}$} & \multirow{2}{*}{0,081} \\
\hline & $\operatorname{Sim}$ & 12 & $74(61)$ & $26(22)$ & & \\
\hline \multirow{2}{*}{$\begin{array}{l}\text { Tempo de sono }<9 \text { horas por } \\
\text { noite }\end{array}$} & Não & 45 & $87(272)$ & $13(42)$ & \multirow{2}{*}{$\begin{array}{l}13,265 \\
(1)\end{array}$} & \multirow{2}{*}{0,000} \\
\hline & Sim & 55 & 77 (289) & $24(93)$ & & \\
\hline \multicolumn{7}{|l|}{ Sonolência diurna excessiva } \\
\hline Não & & 89 & $84(519)$ & $16(99)$ & \multirow{2}{*}{$\begin{array}{l}42,449 \\
\text { (1) }\end{array}$} & \multirow{2}{*}{0,000} \\
\hline Sim & & 11 & $53(40)$ & $47(36)$ & & \\
\hline
\end{tabular}

Tabela 5 - Característica da família e do adolescente e a sua associação com dependência de Internet (analise comparativa)

\begin{tabular}{|c|c|c|c|c|c|}
\hline \multicolumn{2}{|l|}{ Características } & $\begin{array}{c}\text { Sem dependência } \\
\text { Internet } \\
\% \text { (n) / x } \pm \text { SD }\end{array}$ & $\begin{array}{c}\text { Com dependência } \\
\text { Internet } \\
\% \text { (n) / x } \pm \text { SD }\end{array}$ & \multirow[t]{2}{*}{$\begin{array}{c}\chi^{2} \\
\text { (df) } / t \text {-Student }\end{array}$} & \multirow[t]{2}{*}{$p$} \\
\hline \multicolumn{4}{|l|}{ Da família } & & \\
\hline \multirow{2}{*}{ Família de tipologia nuclear } & Não & 79 (131) & $21(35)$ & \multirow{2}{*}{$\begin{array}{l}0,378 \\
(1)\end{array}$} & \multirow{2}{*}{0,539} \\
\hline & Sim & $81(463)$ & $19(101)$ & & \\
\hline \multirow{2}{*}{$\begin{array}{l}\text { Mãe com } 9^{\circ} \text { ano de } \\
\text { escolaridade }\end{array}$} & Não & $81(261)$ & $19(62)$ & \multirow{2}{*}{$\begin{array}{l}0,05 \\
(1)\end{array}$} & \multirow{2}{*}{0,941} \\
\hline & $\operatorname{Sim}$ & $80(303)$ & $20(73)$ & & \\
\hline Idade mãe & & $42,1+/-5,4$ & $42,4+/-5,5$ & $\begin{array}{c}-0,593 \\
(693)\end{array}$ & 0,553 \\
\hline \multirow{2}{*}{ Pai com $9^{\circ}$ ano escolaridade } & Não & $81(281)$ & $19(66)$ & \multirow{2}{*}{$\begin{array}{l}0,094 \\
(1)\end{array}$} & \multirow{2}{*}{0,759} \\
\hline & Sim & $80(277)$ & $20(69)$ & & \\
\hline Idade pai & & $44,5+/-5,6$ & $45,6+/-5,6$ & $\begin{array}{c}-0,059 \\
(687)\end{array}$ & 0,953 \\
\hline \multicolumn{6}{|l|}{ Do adolescente } \\
\hline \multirow{2}{*}{ Género } & Masculino & $84(279)$ & $16(54)$ & \multirow{2}{*}{$\begin{array}{l}3,901 \\
(1)\end{array}$} & \multirow{2}{*}{0,048} \\
\hline & Feminino & 77 (289) & $23(82)$ & & \\
\hline \multirow{2}{*}{$\begin{array}{l}\text { Pratica regular de atividade } \\
\text { física extracurricular }\end{array}$} & Não & 79 (226) & $21(58)$ & \multirow{2}{*}{$\begin{array}{c}0,372 \\
(1)\end{array}$} & \multirow{2}{*}{0,542} \\
\hline & Sim & $83(342)$ & $17(78)$ & & \\
\hline \multirow{2}{*}{$\begin{array}{l}\text { Atividades de grupo } \\
\text { extracurriculares }\end{array}$} & Não & $80(328)$ & $20(87)$ & \multirow{2}{*}{$\begin{array}{l}1,757 \\
(1)\end{array}$} & \multirow{2}{*}{0,185} \\
\hline & Sim & $81(240)$ & $19(49)$ & & \\
\hline \multirow{2}{*}{ Reprovações prévias } & Não & $81(458)$ & $19(106)$ & \multirow{2}{*}{$\begin{array}{l}0,499 \\
(1)\end{array}$} & \multirow{2}{*}{0,480} \\
\hline & Sim & $79(110)$ & $21(30)$ & & \\
\hline
\end{tabular}


tem na rotina dos adolescentes, utilizada diariamente por $75 \%$ da amostra, em que $41 \%$ fê-lo durante três ou mais horas por dia. Por sua vez, o projeto EU Kids online, em que Portugal está integrado, no seu relatório de 2010, revelou que $60 \%$ de um total de 25,142 crianças e adolescentes entre os nove e 16 anos utilizava a Internet diariamente. ${ }^{27}$ Estes dados sugerem uma tendência crescente do uso de Internet e denotam a sua importância no quotidiano dos adolescentes.

Para além de uma utilização mais frequente, os resultados confirmam também a tendência de um início de utilização mais precoce. Aproximadamente metade da amostra fez o seu primeiro acesso até aos oito anos de idade e $28 \%$ admitiu tê-lo realizado até à entrada no primeiro ciclo. De acordo com dados de 2014, a idade média de início de utilização de Internet por parte dos adolescentes portugueses foi de 8,6 anos, similar à de outros países europeus, que variou de 7,9 anos no Reino Unido a 9,5 anos na Itália. ${ }^{28}$ Esta tendência é ainda reforçada se tivermos em consideração dados relativos a 2010 que revelaram uma idade média no primeiro acesso de 10 anos. ${ }^{29}$

Os adolescentes da nossa amostra acederam à Internet maioritariamente em casa, através da utilização do telemóvel e portátil, tal como demostrado no relatório nacional do projeto Net Children Go Mobile, em que, à semelhança de outros países europeus, também em Portugal de 2010 para 2014 se intensificou o acesso no lar, através de dispositivos individuais. ${ }^{27-29}$ Contrariamente ao divulgado nesse relatório, no qual a escola foi apontada como o segundo local de acesso mais frequente, os nossos resultados mostraram que o uso de Internet móvel grátis $(61 \%)$ foi a segunda forma mais comum de acesso, sendo o acesso realizado em meio escolar a quarta opção $(51 \%) .{ }^{29}$ A evolução tecnológica, com melhor e mais vasta oferta de rede wi-fi grátis pode ser apontada como uma possível explicação para este achado. Por outro lado, os resultados obtidos contrapõem também a ideia de que o acesso em movimento, 'em qualquer lugar', que até então parecia não ter expressão entre os adolescentes portugueses, parece ter-se generalizado, uma vez que utilização de Internet móvel, gratuita ou paga, foi usada por $61 \%$ e $34 \%$ dos adolescentes da nossa amostra, respetivamente. ${ }^{29}$ Também os dispositivos utilizados para aceder à Internet, o telemóvel, o portátil e o tablet, reforçam a preferência pelo acesso em movimento, o que torna a utilização de Internet menos passível de controlo parental. Apesar disso, relativamente à supervisão e controlo parental, metade da amostra admitiu seguir com frequência as recomendações parentais em relação à

Tabela 6 - Caracterização da atividade realizada online de acordo com a existência ou não de dependência de Internet (análise comparativa)

\begin{tabular}{|c|c|c|c|c|c|}
\hline Características & & $\begin{array}{c}\text { Sem dependência } \\
\text { Internet } \\
\%(n)\end{array}$ & $\begin{array}{c}\text { Com } \\
\text { dependência Internet } \\
\%(n)\end{array}$ & $\chi^{2}(\mathrm{df})$ & $p$ \\
\hline \multicolumn{6}{|c|}{ Local de acesso à Internet } \\
\hline \multirow{2}{*}{ Casa } & Não & $79(34)$ & $21(9)$ & \multirow{2}{*}{$\begin{array}{c}0,076 \\
(1)\end{array}$} & \multirow{2}{*}{0,782} \\
\hline & $\operatorname{Sim}$ & $81(534)$ & $19(127)$ & & \\
\hline \multirow{2}{*}{ Internet móvel própria } & Não & 85 (394) & $15(72)$ & \multirow{2}{*}{$\begin{array}{c}13,228 \\
(1)\end{array}$} & \multirow{2}{*}{0,000} \\
\hline & $\operatorname{Sim}$ & $73(174)$ & $27(64)$ & & \\
\hline \multirow{2}{*}{ Internet móvel gratuita } & Não & $83(433)$ & $17(86)$ & \multirow{2}{*}{$\begin{array}{c}9,568 \\
(1)\end{array}$} & \multirow{2}{*}{0,002} \\
\hline & $\operatorname{Sim}$ & $73(135)$ & $27(50)$ & & \\
\hline \multirow{2}{*}{ Escola } & Não & $85(210)$ & $15(37)$ & \multirow{2}{*}{$\begin{array}{c}4,595 \\
(1)\end{array}$} & \multirow{2}{*}{0,032} \\
\hline & Sim & $78(358)$ & $22(99)$ & & \\
\hline \multicolumn{6}{|l|}{ Tipo de atividade online } \\
\hline \multirow{2}{*}{ Jogos online } & Não & $84(241)$ & $16(47)$ & \multirow{2}{*}{$\begin{array}{c}2,812 \\
(1)\end{array}$} & \multirow{2}{*}{0,095} \\
\hline & $\operatorname{Sim}$ & $79(327)$ & $21(89)$ & & \\
\hline \multirow{2}{*}{ RS } & Não & $87(138)$ & $13(21)$ & \multirow{2}{*}{$\begin{array}{c}4,920 \\
(1)\end{array}$} & \multirow{2}{*}{0,027} \\
\hline & $\operatorname{Sim}$ & $79(430)$ & $21(11 \%)$ & & \\
\hline \multicolumn{6}{|l|}{ Tipo de RS utilizadas } \\
\hline \multirow{2}{*}{ Facebook } & Não & $82(198)$ & $18(44)$ & \multirow{2}{*}{$\begin{array}{c}0,306 \\
(1)\end{array}$} & \multirow{2}{*}{0,580} \\
\hline & $\operatorname{Sim}$ & $80(370)$ & $20(92)$ & & \\
\hline \multirow{2}{*}{ Instagram } & Não & $86(231)$ & $14(38)$ & \multirow{2}{*}{$\begin{array}{c}7,529 \\
(1)\end{array}$} & \multirow{2}{*}{0,006} \\
\hline & $\operatorname{Sim}$ & $77(337)$ & $23(98)$ & & \\
\hline \multirow{2}{*}{ Snapchat } & Não & $83(271)$ & 17 (59) & \multirow{2}{*}{$\begin{array}{c}2,829 \\
(1)\end{array}$} & \multirow{2}{*}{0,093} \\
\hline & Sim & 78 (297) & $22(22)$ & & \\
\hline \multirow{2}{*}{ Twitter } & Não & 84 (488) & $16(95)$ & \multirow{2}{*}{$\begin{array}{c}19,890 \\
(1)\end{array}$} & \multirow{2}{*}{0,000} \\
\hline & Sim & $66(80)$ & $34(41)$ & & \\
\hline
\end{tabular}


utilização da Internet e a mesma percentagem referiu um bom conhecimento parental das atividades realizadas online. Estes resultados destacam a importância de sensibilizar os pais, os principais pivôs na educação do adolescente, para estas questões, uma vez que a mediação parental é uma ferramenta essencial, quer em termos de gestão do tempo utilizado em atividades online, quer em relação ao tipo de atividades realizadas e à sua segurança. É portanto premente conhecer também o que os adolescentes fazem quando estão online.

A utilização diária de jogos online (34\%) e RS (67\%) na nossa amostra foi superior à reportada pelo Health Behaviour in School - Aged Children, que revelou que 17,4\% dos adolescentes utilizavam as novas tecnologias diariamente para falar com os amigos e $13 \%$ as RS. ${ }^{31}$ Contudo, tendo em consideração os resultados do Net Children Go Mobile, a utilização diária de jogos online (21\%) e RS (50\%), embora menor, foi mais aproximada à da nossa amostra. ${ }^{29}$ No que diz respeito às RS utilizadas, apuramos que $77 \%$ usou o Instagram, $74 \%$ o Facebook, $69 \%$ o Snapchat e $22 \%$ o Twitter. Estes resultados revelam a utilização simultânea de múltiplas RS e o aumento da popularidade do Instagram, uma vez que publicações anteriores mostraram que 97\% dos adolescentes utilizava o Facebook, sendo as restantes RS utilizadas raramente (19\% o Instagram e $2 \%$ o Twitter)..$^{29}$

Em relação à prevalência da dependência de Internet são reportados valores tão díspares como de 1\% na Grécia, $7,9 \%$ na Islândia, $18 \%$ Reino Unido e $22,8 \%$ na Espanha, o que dificulta a interpretação do valor de $19 \%$ obtido na amostra. ${ }^{32,33} \mathrm{O}$ projeto Net Children Go Mobile apurou que na Europa um em cada cinco adolescentes tem pelo menos dois comportamentos associados com o uso excessivo de Internet e que em Portugal 16\% utiliza a Internet de forma excessiva, valor inferior à média europeia (21\%) dos sete países envolvidos no estudo. O valor português foi semelhante aos valores da Dinamarca (25\%), Roménia (24\%) e Irlanda (23\%), tendo sido o mais baixo obtido em Itália $(11 \%)$ e o mais elevado no Reino Unido (29\%). ${ }^{28,29}$ Salientamos que nem sempre é fácil estabelecer e comparar prevalências de dependência de Internet, uma vez que não existe uma definição universalmente aceite, assim como critérios diagnósticos irrefutáveis. Consequentemente, a comparação entre estudos, com diferentes metodologias, deve ser feita cautelosamente. Do conhecimento dos autores, este trata-se do primeiro estudo nacional a determinar a dependência de Internet com base na aplicação do questionário IAT. No futuro poderá ser importante estabelecer um estudo a nível europeu ou mundial, baseado em critérios uniformes, para caracterizar adequadamente esta realidade e permitir monitorizar a sua evolução.

De acordo com os resultados obtidos, o género masculino esteve associado à dependência de Internet. Este é um resultado controverso, uma vez que existem autores que demostram que o género masculino está associado a dependência de Internet e outros negam a diferença entre géneros. ${ }^{33-37} \mathrm{O}$ uso de redes sociais e jogos online foi associado a dependência por outros autores, ${ }^{38}$ contudo os nossos resultados demonstraram apenas associação positiva entre dependência de Internet e uso de RS. Este resultado refuta a ideia inicialmente aceite que a dependência de Internet não seria mais do que dependência de jogos online.

A dependência de Internet e comportamentos associados a uso problemático podem ter uma influência significativa sobre o ciclo vigília-sono. ${ }^{21,39}$ Estudos prévios demonstraram que a utilização excessiva de Internet está associada com a insónia, ${ }^{20,22}$ padrões de sono irregulares e sonolência diurna excessiva, ${ }^{22,24}$ tal como se observou no presente estudo. Um mecanismo que pode ajudar a explicar o impacto negativo do uso excessivo de Internet e hábitos de sono pode ser pela utilização de dispositivos (computador/telemóvel/tablet) ao adormecer, o que condiciona um estado de excitação, interferindo assim com os processos necessários ao normal ciclo sono-vigília. ${ }^{39,40}$ Do conhecimento dos autores, este trata-se do primeiro estudo nacional a determinar a associação positiva com hábitos de sono e sonolência diurna excessiva.

A principal limitação do presente estudo é a utilização de uma amostra de conveniência, o que impossibilita a extrapolação dos resultados para a população, os adolescentes portugueses. Os autores esperam que este estudo constitua um ponto de partida para futuras investigações, para que possamos conhecer o perfil de utilização de Internet dos adolescentes portugueses. A realização de estudos prospetivos permitirá aferir também como evolui essa utilização, assim como as eventuais repercussões na vida dos seus jovens utilizadores.

Em suma, os resultados demonstram a relevância que a Internet tem na rotina dos adolescentes, que priorizam o seu uso no acesso a redes sociais e jogos online, fazendo-o com dispositivos de uso individual, menos passíveis de controlo parental. A dependência de Internet e a sua associação com alterações no sono e sonolência diurna excessiva, enfatiza a relevância desta problemática e a necessidade de sensibilizar os pais, profissionais de educação e profissionais de saúde, para o seu reconhecimento e prevenção. Concluímos ainda que um acesso à Internet mais frequente, realizado em mais locais, através da utilização dispositivos móveis, para realizar mais atividades simultaneamente é o que caracteriza a utilização da Internet por parte dos adolescentes.

\section{CONCLUSÃO}

- A utilização de Internet faz parte da rotina diária os adolescentes, que priorizam o seu uso no acesso a redes sociais e jogos online.

- Apurou-se dependência de Internet em 19\% da amostra, destes $2 \%$ apresentavam critérios de dependência de grave.

- A dependência de Internet está associada a alterações no sono e sonolência diurna excessiva, o que enfatiza a relevância desta problemática. 


\section{AGRADECIMENTOS}

Agradecimento às autoras responsáveis pela validação dos questionários para população portuguesa, Halley M. Pontes e Teresa Moreno. Aos diretores dos agrupamentos de escola que aceitaram colaborar, aos professores e aos alunos.

\section{PROTECÇÃO DE PESSOAS E ANIMAIS}

Os autores declaram que os procedimentos seguidos estavam de acordo com os regulamentos estabelecidos pelos responsáveis da Comissão de Investigação Clínica e Ética e de acordo com a Declaração de Helsínquia da Associação Médica Mundial.

\section{REFERÊNCIAS}

1. Currie C, Zanotti C, Morgan A, Currie D, Looze M, Roberts C, et al. editors. Social determinants of health and well-being among young people. Health Behaviour in School-aged Children (HBSC) study: international report from the 2009/2010 survey. Copenhagen: WHO; 2012.

2. Holstein BE, Pedersen TP, Bendtsen P, Madsen KR, Meilstrup CR, Nielsen L, et al. Perceived problems with computer gaming and Internet use among adolescents: measurement tool for non-clinical survey studies. BMC Public Health. 2014;14:361

3. Tsitsika AK, Andrie EK, Psaltopoulou T, Tzavara CK, Sergentanis TN, Ntanasis-Stathopoulos I, et al. Association between problematic Internet use, socio-demographic variables and obesity among European adolescents. Eur J Public Health. 2016;4:617-22.

4. Stald G, Green L, Barbovski M, Haddon L, Mascheroni G, Ságvári B, et al. Online on the mobile: Internet use on smartphones and associated risks among youth in Europe [eprint]. EU Kids online. 2014 [consultado 2016 ago 10]. Disponível em: http://www.eukidsonline.net.

5. Young KS. Internet addiction: The emergence of a new clinical disorder. Cyberpsychol Behav. 2009;1:237-44.

6. Kwon JH, Chung CS, Lee J. The effects of escape from self and interpersonal relationship on the pathological use of Internet games. Community Ment Health J. 2011;47:113-21.

7. Weinstein A, Lejoyeux M. Internet addiction or excessive Internet use. Am J Drug Alcohol Abuse. 2010;36:277-83.

8. Moreno MA, Jelenchick L, Cox E, Young H, Christakis DA. Problematic Internet use among US youth: a systematic review. Arch Pediatr Adolesc Med. 2011;165:797-805.

9. Frangos CC, Frangos CC, Sotiropoulos I. Problematic Internet use among Greek university students: an ordinal logistic regression with risk factors of negative psychological beliefs, pornographic sites, and online games. Cyberpsychol Behav Soc Netw. 2011;14:51-8.

10. Frangos CC, Frangos CC, Sotiropoulos J. A Meta-analysis of the reliabilty of young's Internet addiction test [eprint]. World Congress on Engineering. 2012. [consultado 2016 ago 12]. Disponível em: http:// www.iaeng.org

11. Widyanto L, Griffiths M. Internet addiction: a critical review. Int J Ment Health Addiction. 2006;4:31-51.

12. Sinkkonen $\mathrm{HM}$, Puhakka $\mathrm{H}$, Meriläinen $\mathrm{M}$. Internet use and addiction among Finnish adolescents (15-19 years). J Adolesc. 2014;37:123-31.

13. Milani L, Osualdella D, Di Blasio P. Quality of interpersonal relationships and problematic Internet use in adolescence. Cyberpsychol Behav. 2009;12:681-4

14. lacovelli A, Valenti S. Internet addiction's effect on likeability and rapport. Comput Hum Behav. 2009;25:439-44.

15. Gackenbach J. Psychology and the Internet: intrapersonal, interpersonal, and transpersonal implications [e-book]. $2^{\mathrm{a}}$ ed. San Diego, Academic Press; 2017 [consultado 2016 jul 30]. Disponível em: http://www. sciencedirect.com

16. Kaess M, Durkee T, Brunner R, Carli V, Parzer P, Wasserman C et al. Pathological Internet use among European adolescents: psychopathology and self-destructive behaviours. Eur Child Adolesc Psychiatry. 2014;23:1093-102.

17. Bener A, Bhugra D. Lifestyle and depressive risk factors associated with problematic Internet use in adolescents in an Arabian Gulf culture. J Addict Med. 2013;7:236-42.

18. Durak M, Senol-Durak E. Associations of social anxiety and depression

\section{CONFIDENCIALIDADE DOS DADOS}

Os autores declaram ter seguido os protocolos do seu centro de trabalho acerca da publicação de dados.

\section{CONFLITOS DE INTERESSE}

Os autores declaram a inexistência de conflitos de interesse na realização do presente trabalho.

\section{FONTES DE FINANCIAMENTO}

Os autores declaram não ter recebido subsídios ou bolsas para a elaboração do artigo.

with cognitions related to problematic Internet use in youths. Edu and Sci J. 2013:19-29.

19. Tonioni F, D'Alessandris L, Lai C, Martinelli D, Corvino S, Vasale M, et al. Internet addiction: hours spent online, behaviors and psychological symptoms. Gen Hosp Psychiatry. 2012;34:80-7.

20. Tan Y, Chen Y, Lu Y, Li L. Exploring associations between problematic Internet use, depressive symptoms and sleep disturbance among Southern Chinese adolescents. Int J Environ Res Public Health. 2016;13:313.

21. Lam LT. Internet gaming addiction, problematic use of the Internet, and sleep problems: a systematic review. Curr Psychiatry Rep. 2014;16:444.

22. Canan F, Yildirim O, Sinani G, Ozturk O, Ustunel T, Ataoglu A. Internet addiction and sleep disturbance symptoms among Turkish high school students. Sleep Biol Rhythms. 2013;11:210-3.

23. Van B. Television viewing, computer game playing, and Internet use and self-reported time to bed and time out of bed in secondary-school children. Sleep. 2004;27:101-4.

24. Choi K, Son H, Park M, Han J, Kim K, Lee B, et al. Internet overuse and excessive daytime sleepiness in adolescents. Psychiatry Clin Neurosci. 2009;63:455-62.

25. Pontes H, Patrão I, Griffiths $\mathrm{M}$. Portuguese validation of the Internet Addiction Test: An empirical study. J Behav Addict. 2014;3:107-14

26. Moreno T. Estudo da sonolência diurna e hábitos de sono numa população escolar dos 11-15 anos - Validação em português da "Pediatric Daytime Sleepiness Scale". Lisboa: FMUL; 2012.

27. Management report EU Kids Online II: enhancing knowledge regarding European children's use, risk and safety online. Deliverable D1.6. EU Kids Online, London, UK. [consultado 2016 ago 16] Disponível em: http://eprints.Ise.ac.uk/42874/1/Management_report_EU_Kids_Online II\%28Isero\%29.pdf

28. Mascheroni G, Ólafsson K. Net Children Go Mobile: risks and opportunities. $2^{\text {a }}$ ed. Milano: Educatt; 2014.

29. Simões J, Ponte C, Ferreira E, Doretto J, Azevedo C. Crianças e meios digitais móveis em Portugal: Resultados nacionais do Projeto Net Children Go Mobile. Lisboa; FCT; 2014.

30. Livingstone S, Haddon L, Görzig A, Ólafsson K. Risks and safety on the Internet: The perspective of European children. Full findings. London: LSE, EU Kids Online; 2011

31. Matos MG, Simões C, Camacho I, Reis M, Simões C, Gomez-Baya D, et al. Em tempo de recessão, os adolescentes portugueses continuam saudáveis e felizes ou são ainda saudáveis mas já não felizes? Arq Med. 2015;29:116-22

32. Tsitsika A, Janikian M, Schoenmakers T, Tzavela E, lafsson K, Wojcik S, et al. Addictive behavior in adolescence: a cross-sectional study in seven European countries. Cyberpsychol Behav Soc Netw. 2014;17:528-35.

33. Durkee T, Kaess M, Carli V, Parzer P, Wasserman C, Floderus B, et al. Prevalence of pathological Internet use among adolescents in Europe: demographic and social factors. Addiction. 2012;107:2210-22.

34. Siomos E, Dafouli D, Braimiotis A, Mouzas D, Angelopoulos V. Internet addiction among Greek adolescent students. Cyberpsychol Behav. 2008;11:653-7.

35. Tsai F, Cheng H, Yeh L, Shih C, Chen C, Yang C, et al. The risk factors of Internet addiction - a survey of university freshmen. Psychiatry Res. 2009;167:294- 9 .

36. Bakken J, Wenzel G, Gotestam G, Johansson A, Oren A. Internet addiction among Norwegian adults: a stratified probability sample study. 
Scand J Psychol. 2008;50:121-7.

37. Tang J, Yu Y, Du Y, Ma Y, Zhang D, Wang J. Prevalence of Internet addiction and its association with stressful life events and psychological symptoms among adolescent Internet users. Addict Behav. 2014;39:744-7.

38. Kormas G, Critselis E, Janikian M, Kafetzis D, Tsitsika A. Risk factors and psychosocial characteristics of potential problematic and problematic
Internet use among adolescents: A cross-sectional study. BMC Public Health. 2011;11:595.

39. Jenaro C, Flores N, Gómez-Vela M, González-Gil F, Caballo C. Problematic Internet and cell-phone use: Psychological, behavioral, and health correlates. Addict Res Theory.2007;15:309-20.

40. Spear LP. The adolescent brain and age-related behavioral manifestations. Neurosci Biobehav Rev. 2000;24:417-63. 